NIST

PUBLICATIONS

\title{
An Algorithm to \\ Position the NIST \\ Advanced Automated Master \\ Angle Calibration System (AAMACS) \\ to the Least Angular Step
}

\author{
David E. Gilsinn
}

W. Tyler Estler

U.S. DEPARTMENT OF COMMERCE

Technology Administration

National Institute of Standards

and Technology

Manufacturing Engineering Laboratory

Gaithersburg, MD 20899

$-Q C$

100

.056

4878

NIST

1992

C. 2 



\section{An Algorithm to \\ Position the NIST \\ Advanced Automated Master \\ Angle Calibration System (AAMACS) \\ to the Least Angular Step}

David E. Gilsinn
W. Tyler Estler

U.S. DEPARTMENT OF COMMERCE

Technology Administration

National Institute of Standards

and Technology

Manufacturing Engineering Laboratory

Gaithersburg, MD 20899

July 1992

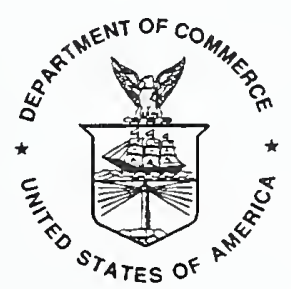

U.S. DEPARTMENT OF COMMERCE Barbara Hackman Franklin, Secretary

TECHNOLOGY ADMINISTRATION

Robert M. White, Under Secretary for Technology

NATIONAL INSTITUTE OF STANDARDS

AND TECHNOLOGY

John W. Lyons, Director 



\section{$\underline{\text { Abstract }}$}

An algorithm based on number theoretic arguments is given that shows how to position the NIST Advanced Automated Master Angle Calibration System (AAMACS) to its least angular step. AAMACS consists of three stacked independently driven serrated tooth indexing tables arranged to rotate concentrically around a common vertical axis. The least angular step between nearest neighbor positions of the topmost table is $\delta \theta_{\min }=$ $2 \pi /(379,080,000) \mathrm{rad}$. The problem of how to select the indexed tooth position of each of the independent tables reduces to the solution of a Diophantine equation in three unknowns. This equation is solved by use of the classic Euclidean Algorithm.

Key words: angle calibration; Euclidean algorithm; Diophantine equations; metrology; number theory; polygon mirrors; small angle measurement 


\subsection{Introduction}

Until recently, the basis techniques for calibrating indexing tables, precision polygon mirrors, and rotary encoders at NIST and other metrology laboratories had not changed greatly in more than thirty years. A series of round-robin intercomparisons involving eight national laboratories demonstrated variability of approximately $\pm 0.2 \operatorname{arc}-\sec ( \pm 1 \mu \mathrm{rad})$ in the measured face-to-face errors of two 12-sided polygon mirrors. This is a typical level of repeatability observed at NIST for precision artifacts with long measurement histories.

In studies supported by the Calibration Coordination Group of the Department of Defense and, more recently, by the Strategic Defense Initiative Organization, we identified a number of factors which conspire to limit the achievable accuracy of angular measurements and calibrations. Principal among these factors are (1) thermal drift associated with manual procedures, (2) refraction and air turbulence effects on small-angle measurements, (3) seismic and acoustic disturbances, and (4) optical errors in autocollimators and polygon mirrors. We have recently installed a new automated angle calibration facility at NIST designed to minimize measurement errors and achieve a factor of ten improvement in fullcircle angle metrology.

Called the Advanced Automated Master Angle Calibration System (AAMACS), the new facility has three major components: (a) a very high resolution indexing table system, (b) a thermally stable, vibration-isolated, custom optical table, and (c) a high-resolution, laser interferometric small-angle measuring system. The heart of the apparatus is a set of three stacked independently driven serrated tooth indexing tables arranged to rotate concentrically around a common vertical axis. This stack is topped by a precision air bearing servo table with integral motor/optical encoder package. The stacked indexing tables form a rotary analog of a differential screw. The tooth numbers of these tables $\left(832=(13)\left(2^{6}\right), 729=3^{6}\right.$, and $625=5^{4}$ respectively) are relatively prime integers, so that the number of discrete angular positions of the topmost table is (832) (729) (625) = $379,080,000$. Conversely, the least angular step between nearest neighbor positions of the topmost table is

$$
\begin{aligned}
\delta \theta_{\min } & =2 \pi /(379,080,000) \mathrm{rad}, \\
& \approx 17 \mathrm{nrad}(0.003 \mathrm{arc}-\mathrm{sec}) .
\end{aligned}
$$

If each tooth is numbered, the object of this note is to determine how to select three integers $\mathrm{x}, \mathrm{y}, \mathrm{z}$ so that

$$
x(2 \pi / 625)+y(2 \pi / 729)+z(2 \pi / 832)=\delta \theta_{\text {min }}
$$


where $2 \pi / 625,2 \pi / 739,2 \pi / 832$ are the smallest angular increments of each of the 3 tables individually. For notation let $N_{1}=625, N_{2}=729$ and $N_{3}=832$. If (1.1) is divided by $2 \pi$, then (1.1) can be rewritten in the form

$$
N_{2} N_{3} x+N_{1} N_{3} y+N_{1} N_{2} Z=1
$$

Both positive and negative integer values for $\mathrm{x}, \mathrm{y}$ and $\mathrm{z}$ are allowed, where a negative value, say for $\mathrm{x}$, will mean stepping a table in a counterclockwise direction $\mathrm{x}$ teeth. A positive value indicates a clockwise turn.

It is clear that (1.2) will lead to negative values for $\mathrm{x}, \mathrm{y}$ or $\mathrm{z}$, if there exists any solution triple. In order to work with positive integers $\mathrm{x}, \mathrm{y}$ and $\mathrm{z}$ we will first look at a slightly different problem. We wish to find the triple of integers $x, y, z$ that will solve

$$
x(2 \pi / 625)+y(2 \pi / 729)+z(2 \pi / 832)=2 \pi+\delta \theta_{\min } .
$$

This means we want to find how many teeth to move each of the tables in order for the topmost table to make one complete rotation plus the smallest increment. This would still give us a relative motion of the topmost table of $\delta \theta_{\min }$.

Using the previous notation, (1.3) can be rewritten as

$$
N_{2} N_{3} x+N_{1} N_{3} y+N_{1} N_{2} z=N_{1} N_{2} N_{3}+1 .
$$

Equations (1.2) and (1.4), where all quantities are integers, are known as Diophantine equations (see [2], p. 96). In this report we will show how elementary number theory can be used to solve them. Of course, the solution could be found by a simple brute force computer search, but the point of this article is to show an interesting application of number theory. Furthermore, this problem has a long history connected with the subject of gear ratios as applied to clocks and other mechanisms.

In section 2.0 we will present the elementary number theory results relevant to the solution of (1.2) or (1.4). In section 3.0 we will solve (1.4) and in section 4.0 we will solve (1.2) and consider some extension questions. 


\subsection{Theoretical Background}

\subsection{Elementary Number Theoretic Properties}

The solution of (1.4) rests on the fact that the division of integers can be written as a quotient plus remainder. It is called the division algorithm, a proof of which can be found in Stewart [2].

The Division Algorithm. Let $a$ be an integer (the dividend) and $b$ a given nonzero integer (the divisor). Then there exist unique integers $q$ (the quotient) and $r$ (the remainder) such that

$$
a=q b+r, \text { and } 0 \leq r<|b| .
$$

The numbers $q$ and $r$ can be found in a finite number of steps.

A closely related concept to the division algorithm is that of the greatest common divisor of two integers $a$ and $b$. If $a$ and $b$ are integers and if there are integers $A, B, d$ such that $a=A d$ and $b=B d$ then $d$ is called a common divisor of $a$ and $b$. Now if $d$ is a common divisor of $a$ and $b$ and every common divisor of $a$ and $b$ is also a divisor of $d$ then $d$ is called a greatest common division of $a$ and $b$ and is designated

$$
d=(a, b)
$$

Furthermore if $a, b, c$ are three integers then the greatest common divisor of $a, b, c$ is designated by

$$
d=(a, b, c)
$$

We note that in $(2.3)$ we also have

$$
d=((a, b), c)=(a,(b, c)) .
$$

If $d=(a, b)$ for integers $a, b$ then $d$ can be represented in terms of $a$ and $b$. In particular, given two nonzero integers $a$ and $b$, their greatest common divisor $d$, which can be selected to be positive, is related to $a$ and $b$ by

$$
(a, b)=d=s a+t b
$$

where $s$ and $t$ are some integers (see [3], p. 18, or [2], p. 35).

In order to find the greatest common divisor of two integers, we use the Euclidean Algorithm (for a proof see [3], p. 18-19). Here we only state the algorithm. 
Euclidean Algorithm. Let $a$ and $b$ be positive integers and suppose $0<b \leq a$. By the division algorithm

$$
a=q b+r, 0 \leq r<b .
$$

If $r=0$, stop, otherwise continue as follows:

$$
\begin{aligned}
b & =q_{1} r+r_{1}, \quad 0 \leq r_{1}<r, \\
r & =q_{2} r_{1}+r_{2}, 0 \leq r_{2}<r_{1}, \\
\cdots & \\
r_{k-2} & =q_{k} r_{k-1}+r_{k}, 0 \leq r_{k}<r_{k-1}, \\
r_{k-1} & =q_{k+1} r_{k},
\end{aligned}
$$

where $b>r>r_{1}>r_{2}>\ldots>r_{\mathrm{k}+1}=0$. The process terminates in a finite number of steps. Then set

$$
d=(a, b)=r_{k}
$$

Next we must introduce the notions of prime and relatively prime integers. An integer $a$ is prime if $a$ is not 0 or +1 or -1 and if $a$ is divisible by $+1,-1,+a,-a$ only. Two integers, $a, b$, are relatively prime if

$$
(a, b)=1
$$

One easy way to check relatively primeness of two integers is to use the fact that any nonzero integer can be factored uniquely into a product of positive primes. The product is multiplied by +1 , or -1 to attach the appropriate sign. Two nonzero integers are relatively prime if they do not have common prime factors. As an example the tooth numbers for the AAMACS tables are $832\left(=(13)\left(2^{6}\right)\right), 729\left(=3^{6}\right)$ and $625\left(=5^{6}\right)$. Since they have no common factors, they are relatively prime.

Finally, as a tool for solving Diophantine equations we introduce the idea of a congruence relation where two integers $a$ and $b$ are said to be congruent modulo an integer $m$ if $m$ divides $a-b$. We will write this as 


$$
a=b(\bmod m)
$$

We will state some algebraic properties here without proofs (for proofs and other properties see [3], pp. 23-25).

P1) If $a=b(\bmod m)$ then for all integers $x$,

$$
\begin{aligned}
a+x & =b+x(\bmod m), \\
a x & =b x(\bmod m), \\
-a & =-b(\bmod m) .
\end{aligned}
$$

P2) If $(c, m)=1$ and

$$
c a=c b(\bmod m)
$$

then

$$
a=b(\bmod m)
$$

This is a cancellation of factors property.

P3) If $(c, m)=1$, then

$$
c x=b(\bmod m)
$$

has an integer solution. This is a division property.

P4) If $(a, m)=d$ then

$$
a x=b(\bmod m)
$$

has no solution when $d$ is not a divisor of $b$. If $d$ divides $b$ there are exactly $d$ solutions. (Stewart [2], p. 92) 


\subsection{Diophantine Equations}

The equations (1.2) and (1.4) are specific cases of the general Diophantine equation

$$
a x+b y+c z=n
$$

where $a, b, c$ and $n$ are integers and the problem is to determine integers $x, y, z$ that satisfy (2.9). The discussion of the solution method for (2.9) is based on [2], pp. 96-99.

We begin with a Diophantine equation in two unknowns

$$
a x+b y=n,
$$

where $a, x, b, y$ and $n$ are integers. As a result of property $\mathbf{P} 4$ in the previous section (2.10) has a solution pair of integers $X$ and $Y$ if and only if $d=(a, b)$ divides $n$. Every solution is given in the form

$$
x=X+B t, y=Y-A t,
$$

where $a=A d$ and $b=B d$ and $t$ is an arbitrary integer.

Now consider (2.9). Write it in the form

$$
a x+b y=n-c z \text {. }
$$

The result above implies that, for a fixed $z,(2.11)$ has integral solutions $X$ and $Y$ if and only if

$$
n-c z=0\left(\bmod d_{1}\right)
$$


where $d_{1}=(a, b)$, or using P1

$$
c z=n\left(\bmod d_{1}\right) .
$$

But from property P4 again (2.13) has an integral solution provided $d=\left(d_{1}, c\right)$ divides $n$. But $d=\left(d_{1}, c\right)=((a, b), c=(a, b, c)$. Therefore (2.9) has a solution triple if and only if $d=(a, b, c)$ divides $n$.

\subsection{Gear Step Solution}

In this section we will show two techniques for solving (1.4). The first makes direct appeals to the results in section 2.0. The second is a somewhat more direct method that does not completely rely on the number theory results in section 2.0 .

\subsection{Number Theoretic Method}

We begin this section by introducing the following notation in (1.4). Let

$$
\begin{aligned}
a & =N_{2} N_{3}, \\
b & =N_{1} N_{3}, \\
c & =N_{1} N_{2} \\
n & =N_{1} N_{2} N_{3}+1 .
\end{aligned}
$$

Then (1.4) becomes

$$
a x+b y+c z=n \text {. }
$$

Write this in the form

$$
a x+b y=n-c z \text {. }
$$


For a fixed $z$ this equation has a solution in integers $x, y$ if and only if $d_{1}=(a, b)$ divides $n-c z$ or in congruence form

$$
n-c z=0\left(\bmod d_{1}\right) \text {. }
$$

Furthermore, every solution in integers $x, y$ is given in the form

$$
\begin{aligned}
& x=X+B t, \\
& y=Y-A t,
\end{aligned}
$$

where $A, B$ are defined by

$$
\begin{aligned}
& a=A d_{1}, \\
& b=B d_{1},
\end{aligned}
$$

and $t$ is an arbritary integer. At this point we need to solve (3.4) or

$$
c z=n\left(\bmod d_{1}\right)
$$

But this can be solved for $z$ under the following conditions:

(1) If $d=\left(c, d_{1}\right)$, then $c z=n\left(\bmod d_{1}\right)$ has no solution when $d$ is not an advisor of $n$.

(2) If $d$ divides $n$, then there are exactly $d$ solutions (i.e., $d$ distinct modulo classes).

In our case we know that $a=N_{2} N_{3}, b=N_{1} N_{3}$ and $\left(N_{2}, N_{1}\right)=1$ so that $d_{1}=\left(N_{2} N_{3}, N_{1} N_{3}\right)$ $=N_{3}$. Furthermore $d=\left(c, d_{1}\right)=\left(N_{1} N_{2}, N_{3}\right)=1$ since $N_{1}, N_{2}, N_{3}$ are relative prime. Then $d$ obviously divides $n=N_{1} N_{2} N_{3}+1$. Thus we can solve (3.7) for $Z$ and all integers of the form

$$
z=Z+N_{3} t_{1}
$$


The selection of an integer $t$, is made in order to satisfy

$$
-N_{3}<z<N_{3}
$$

where $N_{3}$ represents the number of teeth on gear 3 . That is

$$
-N_{3}<Z+N_{3} t_{1}<N_{3}
$$

or

$$
-1-Z / N_{3}<t_{1}<1-Z / N_{3}
$$

For this $z=Z+N_{3} t_{1}$ return to (3.3) to solve for $x$ and $y$.

From (3.1), (3.6) and $d_{1}=N_{3}$ we have

$$
\begin{aligned}
& A=a / d_{1}=N_{2}, \\
& B=b / d_{1}=N_{1} .
\end{aligned}
$$

Therefore from (3.5) all solutions of (3.3) are given by

$$
\begin{aligned}
& x=X+N_{1} t, \\
& y=Y-N_{2} t,
\end{aligned}
$$

for some $t$. Thus we seek an integer $t$ so that both inequalities

$$
\begin{aligned}
& -N_{1}<x<N_{1}, \\
& -N_{2}<y<N_{2},
\end{aligned}
$$


are satisfied or that

$$
\begin{aligned}
& -1-X / N_{1}<t<1-X / N_{1}, \\
& -1+Y / N_{2}<t<1+Y / N_{2},
\end{aligned}
$$

must hold simultaneously.

We now apply this argument to our specific case of the three tables with gear teeth numbers

$$
\begin{aligned}
& N_{1}=625, \\
& N_{2}=729, \\
& N_{3}=832 .
\end{aligned}
$$

Then (1.4) becomes

$$
606528 x+520000 y+455625 z=379080000+1 .
$$

This is in the form (3.2) with

$$
\begin{aligned}
a & =606528, \\
b & =520000, \\
c & =455625, \\
n & =379080001 .
\end{aligned}
$$

First we find $d_{1}=(a, b)=(606528,520000)=832=N_{3}$. Let $d=(d, c)=\left(N_{3}, N_{1} N_{2}\right)=$ 1. Now we must solve

$$
c z=n\left(\bmod d_{1}\right)
$$


or

$$
455625 z=379080001(\bmod 832) .
$$

To do this we use the fact that $\left(d_{1}, c\right)=1$. This means that there exist integers $s$ and $t$ such that

$$
455625 s+832 t=1 .
$$

To compute them we use the Euclidean algorithm.

$\underline{\text { Step } 1}$

Let $a=455625, b=832$. Find $q_{\mathrm{o}}, r_{\mathrm{o}}$ so that

$$
a=q_{o} b+r_{o}, \quad 0<r_{o}<b .
$$

Result: $q_{\mathrm{o}}=547, r_{\mathrm{o}}=521$. Now write

$$
\begin{aligned}
r_{o} & =a-q_{o} b, \\
521 & =a-547 b .
\end{aligned}
$$

\section{$\underline{\text { Step } 2}$}

Find $q_{1}, r_{1}$ so that

$$
b=q_{1} r_{o}+r_{1} \quad, \quad o<r_{1}<r_{o} .
$$

Result: $q_{1}=1, r_{1}=311$. 
Now, use (3.20), and write

$$
\begin{aligned}
r_{1} & =b-q_{1} r_{o}, \\
311 & =b-521, \\
& =b-(a-547 b), \\
311 & =-a+548 b .
\end{aligned}
$$

\section{Step 3}

Find $q_{2}, r_{2}$ so that

$$
r_{o}=q_{2} r_{1}+r_{2}, 0<r_{2}<r_{1} \text {. }
$$

Result: $q_{2}=1, r_{2}=210$. Now, use (3.20) and (3.21), and write

$$
\begin{aligned}
r_{2} & =r_{o}-q_{2} r_{1}, \\
210 & =521-311, \\
& =(a-547 b)-(-a+548 b), \\
210 & =2 a-1095 B .
\end{aligned}
$$

$\underline{\text { Step } 4}$

Find $q_{3}, r_{3}$ so that

$$
r_{1}=q_{3} r_{2}+r_{3}, 0<r_{3}<r_{2} .
$$

Result: $q_{3}=1, r_{3}=101$. Now, use (3.21), (3.22), and write

$$
\begin{aligned}
r_{3} & =r_{1}-q_{3} r_{2}, \\
101 & =311-210, \\
& =(-a+548 b)-(2 a-1095 b), \\
101 & =-3 a+1643 b .
\end{aligned}
$$




\section{$\underline{\text { Step } 5}$}

Find $q_{4}, r_{4}$ so that

$$
r_{2}=q_{4} r_{3}+r_{4}, 0<r_{4}<r_{3} .
$$

Result: $q_{4}=2, r_{4}=8$. Now use, (3.22), (3.23), and write

$$
\begin{aligned}
r_{4} & =r_{2}-q_{4} r_{3}, \\
8 & =210-2(101), \\
& =(2 a-1095 b)-2(-3 a+1643 b), \\
8 & =8 a-4381 b .
\end{aligned}
$$

\section{Step 6}

Find $q_{5}, r_{5}$ so that

$$
r_{3}=q_{5} r_{4}+r_{5}, 0<r_{5}<r_{4} \text {. }
$$

Result: $q_{5}=12, r_{5}=5$. Now, use (3.23), (3.24), and write

$$
\begin{aligned}
r_{5} & =r_{3}-q_{5} r_{4}, \\
5 & =101-12(8), \\
& =(-3 a+1643 b)-12(8 a-4381 b), \\
5 & =-99 a+54215 b
\end{aligned}
$$

\section{$\underline{\text { Step } 7}$}

Find $q_{6}, r_{6}$ so that

$$
r_{4}=q_{6} r_{5}+r_{6}, 0<r_{6}<r_{5} .
$$


Result: $q_{6}=1, r_{6}=3$. Now, use (3.24), (3.25), and write

$$
\begin{aligned}
r_{6} & =r_{4}-q_{6} r_{5} \\
3 & =8-5 \\
& =(8 a-4381 b)-(-99 a+54215 b) \\
3 & =107 a-58596 b
\end{aligned}
$$

\section{$\underline{\text { Step } 8}$}

Fine $q_{7}, r_{7}$ so that

$$
r_{5}=q_{7} r_{6}+r_{7}, 0<r_{7}<r_{6} .
$$

Result: $q_{7}=1, r_{7}=2$. Now, use (3.25), (3.26) and write

$$
\begin{aligned}
r_{7} & =r_{5}-q_{7} r_{6} \\
2 & =5-3 \\
& =(-99 a+54215 b)-(107 a-58596 b) \\
2 & =-206 a+112811 b .
\end{aligned}
$$

\section{Step 9}

Find $q_{8}, r_{8}$ so that

$$
r_{6}=q_{8} r_{7}+r_{8}, 0<r_{8}<r_{7} .
$$

Result: $q_{8}=1, r_{8}=1$. Now, use (3.26), (3.27) and write

$$
\begin{aligned}
r_{8} & =r_{6}-q_{8} r_{7}, \\
1 & =3-2, \\
& =(107 a+58596 b)-(-206 a-112811 b), \\
1 & =313 a-171407 b .
\end{aligned}
$$


This step is as far as we need to go to solve (3.19) since in this case we can take

$$
\begin{aligned}
s & =313, \\
t & =-171407,
\end{aligned}
$$

in (3.19).

The final Euclidean algorithm steps yield.

$\underline{\text { Step } 10}$

Find $q_{9}, r_{9}$ so that

$$
r_{7}=q_{9} r_{8}+r_{9}, \quad 0<r_{9}<r_{8}
$$

Result: $q_{9}=1, r_{9}=1$.

Step 11

Find $q_{10}, r_{10}$ so that

$$
r_{8}=q_{10} r_{9}+r_{10}, 0<r_{10}<r_{9}
$$

Result: $q_{10}=1, r_{10}=0$.

This terminates the Euclidean algorithm and confirms that $a$ and $b$ are relatively prime as we also knew from a prime factor decomposition. That is

$$
1=q_{10}=(a, b)
$$

We can now solve (3.18) using (3.28). Multiply (3.28) by 379080001 and get

$$
379080001=a[(313)(379080001)]+b[(-171407)(379080001)]
$$


or

$$
379080001=a(118652040313)+b(-64976965731407) .
$$

This result yields the solution for (3.18) as

$$
Z=118652040313 .
$$

We also have

$$
\begin{aligned}
d_{1} & =N_{3}=832, \\
d & =(832,455625)=1 .
\end{aligned}
$$

Therefore, we can write all solutions of (3.18) in the form (3.8) or

$$
\begin{aligned}
z & =Z+N_{3} t_{1}, \\
& =118652040313+832 t_{1} .
\end{aligned}
$$

We finally seek a value for $t_{1}$, so that $0<z<832$. There is only one integer value for $t_{1}$ that satisfies the constraint and that is

$$
t_{1}=-142610625 \text {. }
$$

This yields the solution for $z$ as

$$
z=313 .
$$


Having found $z$ we put (3.16) in the form (3.3) and get

$$
606528 x+520000 y=236469376
$$

We note that $(606528,520000)=832$. Since $236469376=(284218)(832)$ we can say that (3.30) has a solution pair of integers $X, Y$ and every solution is given by

$$
\begin{aligned}
& x=X+B t, \\
& y=Y-A t,
\end{aligned}
$$

where

$$
\begin{aligned}
& A=606528 / 832=729 \\
& B=520000 / 832=625 .
\end{aligned}
$$

Using (2.2) we look for integers $s$ and $r$ so that

$$
832=606528 s+520000 r .
$$

Again, we apply the Euclidean algorithm technique. Set

$$
\begin{aligned}
& a=606528, \\
& b=520000 .
\end{aligned}
$$

\section{$\underline{\text { Step } 1}$}

Find $q_{o}, r_{\mathrm{o}}$ so that

$$
a=q_{o} b+r_{o}, 0<r_{o}<b .
$$


Result: $q_{\mathrm{o}},=1, r_{\mathrm{o}}=86528$. Now write

$$
r_{o}=a-q_{o} b=a-b .
$$

\section{$\underline{\text { Step } 2}$}

Find $q_{1}, r_{1}$ so that

$$
b=q_{1} r_{o}+r_{1}, 0<r_{1}<r_{o} .
$$

Result: $q_{1}=6, r_{1}=832$. Now write, using (3.33)

$$
\begin{aligned}
r_{1} & =b-q_{1} r_{o} \\
832 & =b-6(86528), \\
& =b-6(a-b), \\
832 & =-6 a+7 b .
\end{aligned}
$$

Therefore (3.32) has a solution

$$
\begin{aligned}
& s=-6, \\
& r=7 .
\end{aligned}
$$

To find $X, Y$ for (3.30), multiply (3.34) by 284218 and get

$$
\begin{aligned}
236469376=a( & -1705308) \\
& +b(1989526)
\end{aligned}
$$

from which we have the particular solutions

$$
\begin{aligned}
X & =-1705308 \\
Y & =1989526
\end{aligned}
$$


Finally we need to find a fixed $t$ so that

$$
\begin{aligned}
& -N_{1}<X+B t<N_{1}, \\
& -N_{1}<Y-A t<N_{2},
\end{aligned}
$$

if it exists. These inequalities are

$$
\begin{aligned}
& -625<-1705308+625 t<625 \\
& -729<1989526-729 t<729 .
\end{aligned}
$$

In fact there is only one integer satisfying both inequalites and is given by

$$
t=2729 .
$$

The final $x, y$ solution sought is then

$$
\begin{aligned}
& x=X+B t=317 \\
& y=Y-A t=85 .
\end{aligned}
$$

Combining (3.37) with (3.29) gives the solution of (3.16) as $x=317, y=85, z=313$. This says that to position the topmost table to the smallest angular increment $\delta \theta_{\min }$, set all tables to their reference 0 mark. Then move the largest table clockwise 313 teeth from the reference, the middle table 85 teeth clockwise and finally the topmost table 317 teeth. The end result will be to move the topmost table $2 \pi+\delta \theta_{\min }$ radius, which is equivalent to moving it $\delta \theta_{\min }$.

\subsection{Constraint Method}

The first steps of this method, proposed by the second author, still require proceeding through the Euclidean Algorithm to obtain $z=313$ in (3.29) and finally (3.30). At this point divide (3.30) by $N_{3}=832$ to obtain the Diophantine equation

$$
729 x+625 y=284218
$$


to be solved for integers $x, y$ subject to the constraints

$$
\begin{aligned}
& -625<x<625 \\
& -729<y<729 .
\end{aligned}
$$

First, rewrite (3.38) as

$$
\begin{aligned}
625 y & =-729 x+B, \\
& =-625 x-104 x+B,
\end{aligned}
$$

where

$$
B=284218 \text {. }
$$

Then, from (3.40) write

$$
y=-x+(B-104 x) / 625 \text {. }
$$

Since $x$ and $y$ are integers any solution of (3.42) requires that there be an integer $r_{1}$ such that

$$
\begin{aligned}
B-104 x & =r_{1}(625), \\
& =(624+1) r_{1} \\
& =6(104) r_{1}+r_{1}
\end{aligned}
$$

or

$$
x=-6 r_{1}+\left(B-r_{1}\right) / 104 \text {. }
$$


Again, since $x, r_{1}$ are to be integers then there must exist an integer $r_{2}$ such that

$$
B-r_{1}=104 r_{2}
$$

Now we use constraint (3.39a) and (3.43) to bound $r_{1}$. That is, if we set

$$
-625<-6 r_{1}+\left(B-r_{1}\right) / 104<625
$$

then

$$
(B / 625)-104<r_{1}<(B / 625)+104
$$

Using (3.41), this inequality gives a bound on $r_{1}$ of

$$
350.7<r_{1}<558.8
$$

This is too wide a range to uniquely select $r_{1}$ so we must estimate $r_{2}$ from (3.44) and (3.45)

$$
-1+(B / 104)(624 / 625)<r_{2}<(B / 104)(624 / 625)+1
$$

or

$$
2727.4<r_{2}<2729.5
$$

There are only two integers satisfying this last inequality, i.e., $r_{2}=2728$ and $r_{2}=2729$. First, let $r_{2}=2729$. Then from (3.44) $r_{1}=402$ and from (3.43) and (3.42)

$$
\begin{aligned}
& x=317, \\
& y=85,
\end{aligned}
$$


as obtained in section 3.1. Next let $r_{2}=2728$. Then $r_{1}=506$ and

$$
\begin{aligned}
& x=-308 \\
& y=814
\end{aligned}
$$

Although $y$ falls out of the range $-729<y<729$ we can interpret the triple

$$
\begin{aligned}
& x=-308 \\
& y=814 \\
& z=313
\end{aligned}
$$

First note that $308+317=625$. This $x=-308$ moves the 625 tooth table counterclockwise 308 teeth. This would position it at the same tooth as $x=317$. Finally, note that $814=729+85$. Setting $y=814$ just rotates the 729 tooth table one complete revolution plus 85 teeth. So, although this is not a formal solution satisfying the constraints, it can be interpreted physically. Since the physical table has stops to prevent the individual toothed gears from rotating more than one revolution in the clockwise or counterclockwise direction this second solution is not physically feasible and thus must be disallowed.

\subsection{Related Problems}

The first problem that we will consider is whether (1.2) has a solution. In this case, we again have $N_{1}=625, N_{3}=832,(1.2)$ becomes

$$
606528 x+520000 y+455625 z=1 .
$$

Now set $a=606528, b=520000, c=455625$ and $n=1$. Then we have (2.7)

$$
a x+b y=n-c z \text {. }
$$


Since $d_{1}=(a, b)=N_{3}=832$ then we look for a $z$ that

$$
c z=1(\bmod 832)
$$

but this has a solution provided $d=\left(c, d_{1}\right)=\left(N_{1} N_{2}, N_{3}\right)=1$ divides $n=1$. Therefore, (4.3) can be solved for $Z$ and all integers of the form

$$
z=Z+832 t_{1}
$$

where $z$ must satisfy

$$
-832<z<832
$$

But, from (3.28), $Z=313$ which implies that $t_{1}=0$ or -1 . If $t_{1}=-1$ then $z=-519$ and (4.1) yields

$$
606528 x+520000 y=236469376 \text {. }
$$

Multiplying (3.34) by 284218 one finds

$$
\begin{aligned}
X & =-1705308 \\
Y & =1989526 .
\end{aligned}
$$

Using (3.12) and 3.13) it is possible to show that there is no value $t$ for which (3.13) is satisfied. Therefore $t_{1}$, cannot be -1 . Set $t_{1}=0$ then $z=313$. From (4.1)

$$
606528 x+520000 y=-142610624 \text {. }
$$


Multiplying (3.34) by -171407 one finds

$$
\begin{aligned}
& x=1028442, \\
& y=-1199849 .
\end{aligned}
$$

From (4.9), (3.12) and (3.13) we look for an integer $t$ that satisfies

$$
\begin{aligned}
& -625<1028442+625 t<625 \\
& -729<-1199849-729 t<729 .
\end{aligned}
$$

These can be solved for a single integer $t$. In fact $t=-1645$. Then

$$
\begin{aligned}
x= & 317 \\
y= & -644
\end{aligned}
$$

We note that $y=-644$ means that the 729 toothed table is moved counterclockwise 644 teeth which would position it at the same tooth as setting $y=85$. Therefore, this is the complementary solution and problem (1.2) is solvable.

A natural question arises. Are there legitimate settings of the indexed tables that will move the topmost table two or more full revolutions plus $\delta \theta_{\text {min }}$. A complete examination of this question is beyond the scope of this report but we will show that the answer is no for the case of $2+\delta \theta_{\min }$ revolutions.

As before $N_{1}=625, N_{2}=729, N_{3}=832$ and (3.2) becomes

$$
N_{2} N_{3} x+N_{1} N_{3} y+N_{1} N_{2} z=2\left(N_{1} N_{2} N_{3}\right)+1
$$


Next we form

$$
N_{2} N_{3} x+N_{1} N_{3} y=2\left(N_{1} N_{2} N_{3}\right)+1-N_{1} N_{2} z
$$

(4.12) has a solution $x, y$ if and only if $d_{1}=\left(N_{2} N_{3}, N_{1} N_{3}\right)=N_{3}=832$ divides $2\left(N_{1} N_{2} N_{3}\right)$ $+1-N_{1} N_{2} z$, or

$$
N_{1} N_{2} z=2\left(N_{1} N_{2} N_{3}\right)+1\left(\bmod N_{3}\right)
$$

or

$$
455625 z=75816001(\bmod 832)
$$

To solve this we note that $(455625,832)=1$ so that we can solve for integers $s$ and $t$ such that

$$
455625 x+832 t=1 \text {. }
$$

But (4.15) is again (3.19) so that

$$
\begin{aligned}
& s=313 \\
& t=-171407 .
\end{aligned}
$$

Now we multiply both sides of (4.15) by 758160001 and get

$$
455625((313)(758160001)+832((-171407)(758160001))=758160001
$$

and get 


$$
455625(237304080313)+832(-129953931291407)=758160001 .
$$

Thus a particular solution for $Z$ in (4.14) is given by

$$
Z=237304080313
$$

Since $d_{1}=N_{3}=832$ and $d=(832,455625)=1$ we can write all solutions of $(4.14)$ in the form

$$
\begin{aligned}
z & =Z+N_{3} t_{1}, \\
& =237304080313+832 t_{1} .
\end{aligned}
$$

We now look for an integer $t_{1}$ so that

$$
-832<237304080313+832 t_{1}<832
$$

An integer solution of this inequality is given by

$$
t_{1}=-285221250
$$

From (4.19)

$$
z=313
$$

which again is the same as previous results. Substituting this into (4.12) we see that we must solve

$$
606528 x+520000 y=615549376
$$

for $-625<x<625,-279<y<729$. But $615549376=(832)(739843)$ so that we can look 
for a solution of

$$
606528 s+520000 r=832 .
$$

But from (3.34) this is $s=-6, r=7$. Multiply (4.24) by 739843 gives

$$
\begin{aligned}
& x=-4439058 \\
& y=5178901
\end{aligned}
$$

Using (3.12) and (3.13) one needs to solve for $t$ such that

$$
\begin{gathered}
-625<-4439058+625 t<625 \\
-729<5178901-729 t<729 .
\end{gathered}
$$

But we see that (4.26a) yields the potential candidate $t=7102$ and (4.26b) yields a different potential candidate 7104 . Since there is no common $t$ value satisfying both inequalities this problem is not solvable.

As a final note, we see that once a procedure for $\delta \theta_{\min }$ has been determined the required tooth address for any final angular position is determined. 


\subsection{References}

1) Estler, W. T., Queen, Y. H., Gilsinn, D., Pieczulewski, D., "Advanced Angle Metrology at the National Institute of Standards and Technology," ASPE Conf., Santa Fe, NM, October, 1991.

2) Stewart, B. M., "Theory of Numbers," The MacMillan Company, New York (1964).

3) Birkhoff, G., MacLane, S., "A Survey of Modern Algebra," The MacMillan Company, New York (1953). 


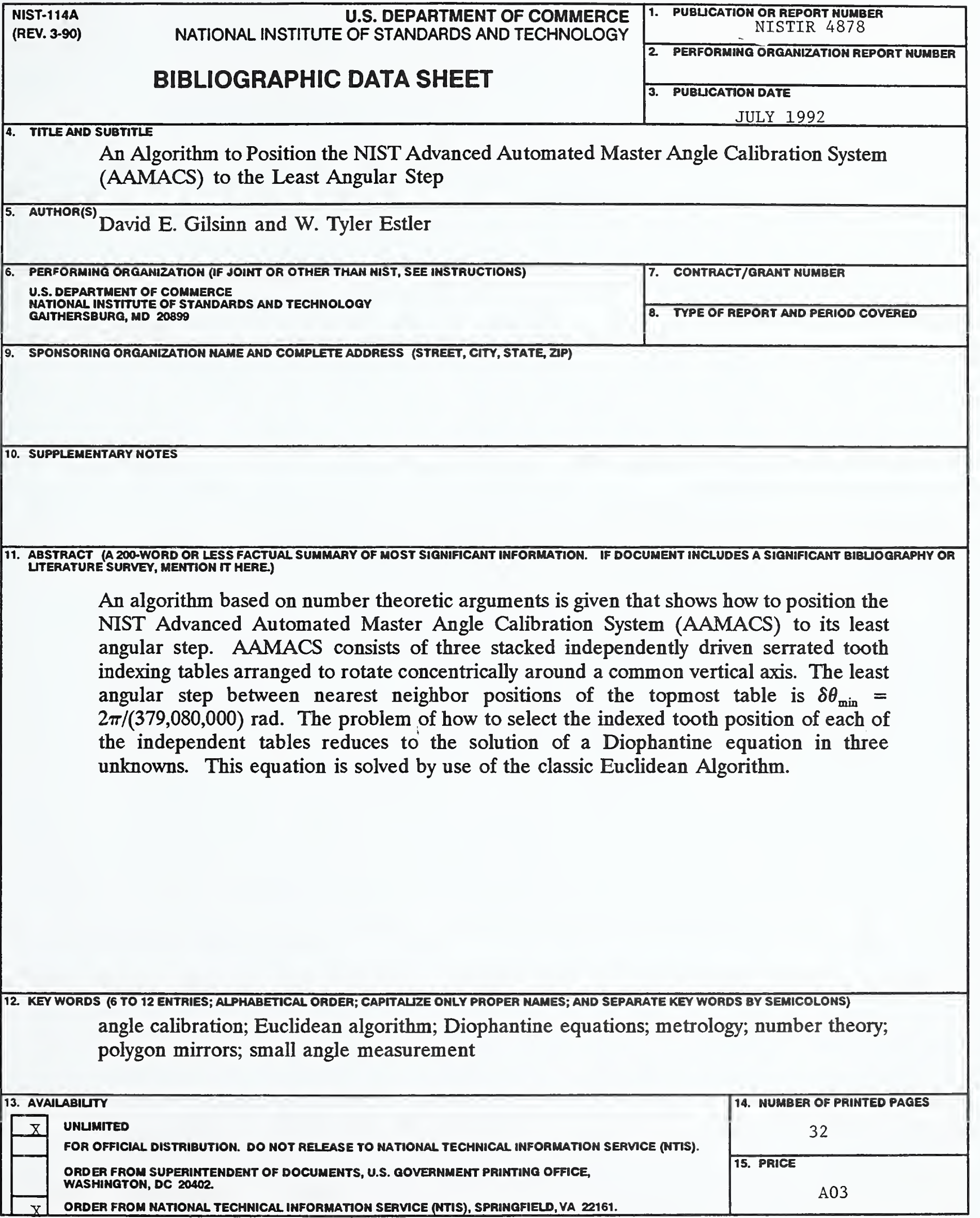



